\title{
Nuevas aportaciones sobre la cronología de los restos conservados de las murallas medievales de Almería (España)
}

New contributions on the chronology of the preserved remains of the medieval city walls of Almeria (Spain)

\section{Antonio Orihuela Uzal}

Laboratorio de Arqueología y Arquitectura de la Ciudad - Escuela de Estudios Árabes - Consejo Superior de Investigaciones Científicas, Granada, Spain, orihuela@eea.csic.es

\begin{abstract}
The medieval city walls of Almeria have abundant references in Arabic sources and numerous preserved remains, either in all its elevation, or as small archaeological remains on the current slope and even under the ground. This circumstance has given rise to a lot of scientific literature on the chronology of each of the different existing precincts: Alcazaba, Medina, suburbs and outer enclosure. The problem lies in the fact that, since its foundation in the tenth century until the conquest by the Catholic Monarchs in 1489 and its reuse until the mid-nineteenth century, the medieval walls have undergone various repairs, extensions and reconstructions. In order to provide greater chronological precision, from the School of Arab Studies (CSIC), a Project of the State Research Plan was requested, which was granted with reference HAR2015-71609-P. It has allowed to make radiocarbon dating of wood and other building materials of the walls, in combination with studies of construction, metrological, historical techniques and restorations carried out since the mid-twentieth century. All this has allowed us to contribute new hypotheses about the chronology of the preserved remains, many of which are much more recent than the foundational walls that they have replaced.
\end{abstract}

Keywords: Radiocarbon, Almeria, medieval city walls, restoration.

\section{Introducción}

Gracias tanto a la abundancia de fuentes árabes como a los numerosos restos conservados, las murallas medievales de Almería han sido objeto de importantes investigaciones desde mediado el siglo XX. Autores clásicos y veteranos como L. Torres (1957), J. A. Tapia (1986), E. Molina (1986), L. Cara (1990), J. Lirola (1992-1993, 2005) y A. Gil (2014), desde los campos de la historia, la arqueología y la filología árabe, además de otros más jóvenes como P. Gurriarán y S. Márquez (2009), y D. Garzón (2015), desde la arquitectura, el urbanismo y la restauración monumental, han hecho importantes aportaciones para el conocimiento de la cronología y la evolución de las diferentes cercas de la ciudad. Por otra parte, hay una importante colección de croquis y planos históricos que recogen la situación de las murallas antes del inicio de su demolición parcial a partir del año 1855: Caffaro (1146), J. de Oviedo (1621), H. Toreli (1694), Ph. Crame (1740), F. Guillamas (1842) y J. Prats (1852), entre otros muchos.

Varios de estos autores han planteado hipótesis muy diversas referentes tanto a la cronología de 
las murallas conservadas como a la datación y finalidad del casi desaparecido recinto exterior, situado al noroeste del Cerro del San Cristóbal. Con el objetivo de aportar una mayor precisión cronológica sobre las murallas conservadas, solicité desde la Escuela de Estudios Árabes (CSIC) un Proyecto del Plan Estatal I+D+i, titulado "Las murallas medievales de Almería, estudio cronotipológico y datación científica", que fue financiado con la referencia HAR201571609-P y ha concluido a fines de 2019. Se han analizado mediante datación por radiocarbono o carbono-14 $\left({ }^{14} \mathrm{C}\right)$ dos grupos de muestras: de morteros de cal y de materias orgánicas como la madera, la soga de fibra vegetal y el carbón vegetal. Además, se ha levantado planimetría fotogramétrica de la muralla del Cerro de San Cristóbal y se han analizado y medido todos los restos de murallas medievales conservados.

\section{Las fortalezas y debilidades de la datación por radiocarbono}

Los análisis de las muestras fueron realizados en el Servicio de Datación por Radiocarbono del CNA (Centro Nacional de Aceleradores), centro mixto de la Universidad de Sevilla, la Junta de Andalucía y el CSIC. El sistema utilizado fue el llamado MiCaDaS (Mini radioCarbon Dating System), que se basa en la técnica de la Espectrometría de Masas con Acelerador (AMS, del inglés Accelerator Mass Spectrometry), controlado por los técnicos F. J. Santos e I. Gómez, quienes elaboraron los correspondientes Informes de Datación y Calibración de cada muestra.

El método de datación por radiocarbono empezó a utilizarse en la década de 1950 y se basa en la ley de decaimiento exponencial del isótopo radiactivo ${ }^{14} \mathrm{C}$, que se genera en la atmósfera como consecuencia del bombardeo de átomos de nitrógeno por neutrones producidos por los rayos cósmicos al interaccionar con la atmósfera. Este isótopo se encuentra homogéneamente mezclado con los átomos estables y no radiactivos del carbono $\left({ }^{12} \mathrm{C}\right.$ y $\left.{ }^{13} \mathrm{C}\right)$ que están combinados químicamente con el oxígeno en forma de $\mathrm{CO}_{2}$. Posteriormente la fotosíntesis incorpora el ${ }^{14} \mathrm{C}$ en las plantas, manteniendo una proporción ${ }^{14} \mathrm{C} /{ }^{12} \mathrm{C}$ en éstas igual a la atmosférica, hasta el momento de su corte, cuando deja de incorporarse y la concentración del isótopo va decreciendo conforme se transforma en ${ }^{14} \mathrm{~N}$ por desintegración radiactiva. Por consiguiente, midiendo la cantidad de ${ }^{14} \mathrm{C}$ que aún queda en una muestra de madera se puede datar el momento del corte del árbol. Esto se conoce como Edad de Radiocarbono convencional (ERC) y se expresa en años BP (Before Present), estableciéndose por convenio como Presente el año 1950 de nuestro calendario (AD), porque en la segunda mitad del siglo $\mathrm{XX}$ los numerosos ensayos nucleares provocaron grandes anomalías en las curvas de concentración relativa de los isótopos radiactivos en la atmósfera. Una vez calculada la edad de la muestra en años BP, se obtiene la edad cronológica mediante la correspondiente curva de calibración, habiéndose utilizado para ello muestras de maderas de edades conocidas por dendrocronología. La edad así hallada se denomina "edad calibrada" y se expresa en años Cal BP (Alejandre, 2018).

También se pueden analizar los morteros de cal, ya que durante su proceso de carbonatación absorben $\mathrm{CO}_{2}$ atmosférico, incorporando el ${ }^{14} \mathrm{C}$ presente en la atmósfera, registrando el tiempo de construcción de un elemento. No obstante, hay una serie de problemas al analizar morteros de cal, debidos principalmente a las arenas calcáreas y a los residuos de calizas por una incompleta cocción de la piedra caliza de la cual se ha obtenido la cal, etc. Estos carbonatos primarios o geológicos, pueden provocar importantes errores de datación, dando como resultado edades cronológicas mucho mayores que las del proceso de fraguado o carbonatación del mortero (Alejandre, 2018).

Otro problema de la datación por radiocarbono aplicado a la baja Edad Media es que la curva de calibración tiene varios puntos de inflexión que producen sus correspondientes picos y zonas bastante horizontales. Por consiguiente, en este periodo histórico se pueden producir varios cruces discontinuos entre la distribución gaussiana de la Edad de Radiocarbono convencional (que suele tener una amplitud de \pm 25 años BP o ligeramente mayor) y la curva de calibración, que darán como resultados otros tantos conjuntos de fechas con sus correspondientes probabi- 
lidades. La decisión sobre cuál es el rango más adecuado puede hacerse basándose en evidencias históricas $\mathrm{u}$ otros datos de medidas, que permitan descartar alguno de ellos (Santos, 2019).

\section{Análisis de los resultados de las muestras datadas}

Se analizaron ocho muestras de costras de tapia calicostrada y morteros de cal de las murallas del Cerro de San Cristóbal. Por las razones expuestas en el apartado anterior, probablemente por contaminación de áridos calizos, los resultados obtenidos dieron dataciones mucho más antiguas que lo que las fuentes históricas permiten esperar, por lo que fueron considerados erróneos y descartados.

Posteriormente se tomaron y analizaron otras once muestras de materiales orgánicos: nueve de madera, una de soga de fibra vegetal y una de carbón vegetal. A continuación, se ofrece una tabla que contiene el resumen de los datos de las muestras y los resultados obtenidos:

\begin{tabular}{|c|c|c|c|c|c|c|}
\hline Muestra & $\begin{array}{l}\text { Código } \\
\text { CNA }\end{array}$ & Material & Ubicación & $\begin{array}{c}\text { Edad } \\
\text { Calibrada AD }\end{array}$ & $\begin{array}{c}\text { Probabilidad } \\
\%\end{array}$ & $\begin{array}{l}\text { Periodo } \\
\text { histórico }\end{array}$ \\
\hline M-10 & 4688.1 .1 & $\begin{array}{ll}\begin{array}{l}\text { Dintel } \\
\text { aspillera }\end{array} & \mathrm{de} \\
\end{array}$ & S.C.-Torre 6 & $1028-1166$ & 100 & $\begin{array}{l}\text { Taifa- Almorá- } \\
\text { vide }\end{array}$ \\
\hline M-11 & 5098.1 .1 & Tabla madera & S.C.-Torre 7 & Moderna & 100 & S. XVII-XX \\
\hline $\mathrm{M}-12$ & 5099.1 .1 & Aguja tapia & S.C.-Torre 6 & $\begin{array}{l}995-1054 \\
1078-1153\end{array}$ & $\begin{array}{l}50 \\
50\end{array}$ & $\begin{array}{l}\text { Califal-Taifa } \\
\text { Almorávide }\end{array}$ \\
\hline M-13 & 5100.1 .1 & Aguja tapia & S.C.-Lienzo 4-5 & $\begin{array}{l}1266-1307 \\
1362-1385\end{array}$ & $\begin{array}{l}79 \\
21\end{array}$ & $\begin{array}{l}\text { Nazarí } \\
\text { Nazarí }\end{array}$ \\
\hline M-14 & 5101.1 .1 & Aguja tapia & S.C.-Torre 4 & $971-1040$ & 100 & Califal-Taifa \\
\hline M-15 & 5102.1 .1 & Aguja tapia & S.C.-Torre 4 & $\begin{array}{l}1046-1091 \\
1121-1140 \\
1147-1225\end{array}$ & $\begin{array}{l}16 \\
4 \\
79\end{array}$ & $\begin{array}{l}\text { Taifa } \\
\text { Almorávide } \\
\text { Almohade }\end{array}$ \\
\hline M-16 & 5103.1 .1 & Aguja tapia & S.C.-Lienzo 2-3 & $\begin{array}{l}1051-1082 \\
1151-1253\end{array}$ & $\begin{array}{l}8 \\
91\end{array}$ & $\begin{array}{l}\text { Taifa } \\
\text { Almohade }\end{array}$ \\
\hline M-17 & 5104.1.1 & Cuña de tapia & Medina-Torre 1 & $\begin{array}{l}1278-1320 \\
1350-1391\end{array}$ & $\begin{array}{l}50 \\
50\end{array}$ & $\begin{array}{l}\text { Nazarí } \\
\text { Nazarí }\end{array}$ \\
\hline M-18 & 5105.1.1 & $\begin{array}{l}\text { Soga fibra } \\
\text { vegetal }\end{array}$ & Medina-Torre 1 & $\begin{array}{l}1299-1369 \\
1380-1411\end{array}$ & $\begin{array}{l}73 \\
27\end{array}$ & $\begin{array}{l}\text { Nazarí } \\
\text { Nazarí }\end{array}$ \\
\hline M-19 & 5106.1 .1 & $\begin{array}{l}\text { Carbón } \\
\text { vegetal }\end{array}$ & Medina-Torre 3 & $\begin{array}{l}648-713 \\
745-764\end{array}$ & $\begin{array}{l}88 \\
12\end{array}$ & $\begin{array}{l}\text { Visigodo } \\
\text { Emiral }\end{array}$ \\
\hline M-20 & 5107.1 .1 & Aguja tapia & Medina-Torre 1 & $\begin{array}{l}1258-1300 \\
1369-1381\end{array}$ & $\begin{array}{l}96 \\
4\end{array}$ & $\begin{array}{l}\text { Nazarí } \\
\text { Nazarí }\end{array}$ \\
\hline
\end{tabular}

Como se puede observar en la tabla precedente, hay resultados de dos muestras que son incoherentes y, por consiguiente, serán descartadas para evitar conclusiones erróneas:
- La muestra M-11 produce una datación moderna, con una edad de radiocarbono convencional $150 \pm 25 \mathrm{BP}$, un periodo demasiado reciente, para el cual esta técnica de datación resulta inadecua- 
da. Es posible que se deba a un fallo en la recogida de la madera, pues en ese lugar hubo construcciones modernas adosadas extramuros a la muralla y puede que al intentar extraer madera de una aguja de la tapia medieval se sacara en realidad una madera moderna introducida en el mechinal de la primitiva aguja.

- La muestra M-19, correspondiente a pequeños nódulos de carbón vegetal existentes en el interior de las tapias de la torre de la muralla de la Medina conservada en la Calle Socorro, ha dado como resultado una datación entre los siglos VII-VIII, que puede deberse a dos razones: Reutilización de una madera cortada en ese periodo o, probablemente, contaminación de la muestra durante su dificultosa extracción con una pequeña proporción de granos de arena caliza natural, que han provocado el retraso en la cronología que se podía esperar por las fuentes documentales históricas.

Los resultados de las seis muestras válidas correspondientes a la muralla del Arrabal del
Oratorio (Rabạ al-Mușallā) en el sector del cerro de San Cristóbal (M-10, M-12, M-13, M14, M-15 y M-16) dan un amplio abanico de cronologías posibles correspondientes a los periodos taifa, almorávide, almohade y nazarí. No obstante, los porcentajes de probabilidades de cada periodo, pueden ayudar algo a clarificar la datación. El geógrafo almeriense al-'Udirī, que vivió en la ciudad en el siglo XI, ha atribuido la construcción fundacional de esta muralla a los inicios del periodo taifa, en concreto a la etapa del emir Jayrān (r. 1014-1028) (Sánchez, 19751976, pp. 36-37). Sin embargo, las dos muestras analizadas de la torre $\mathrm{n}^{\circ} 6$ (contadas de oeste a este empezando desde la esquina occidental del citado cerro) nos dan opciones del periodo taifa, pero también del almorávide en una importante proporción. Todo ello en un tramo en pendiente que va desde el lienzo 5-6 hasta la torre 9 construido con tapia calicostrada de gran calidad, con altura de cajones de tapia de $80-81 \mathrm{~cm}$, y ausencia de reparaciones relevantes en su cara extramuros (Fig. 1).

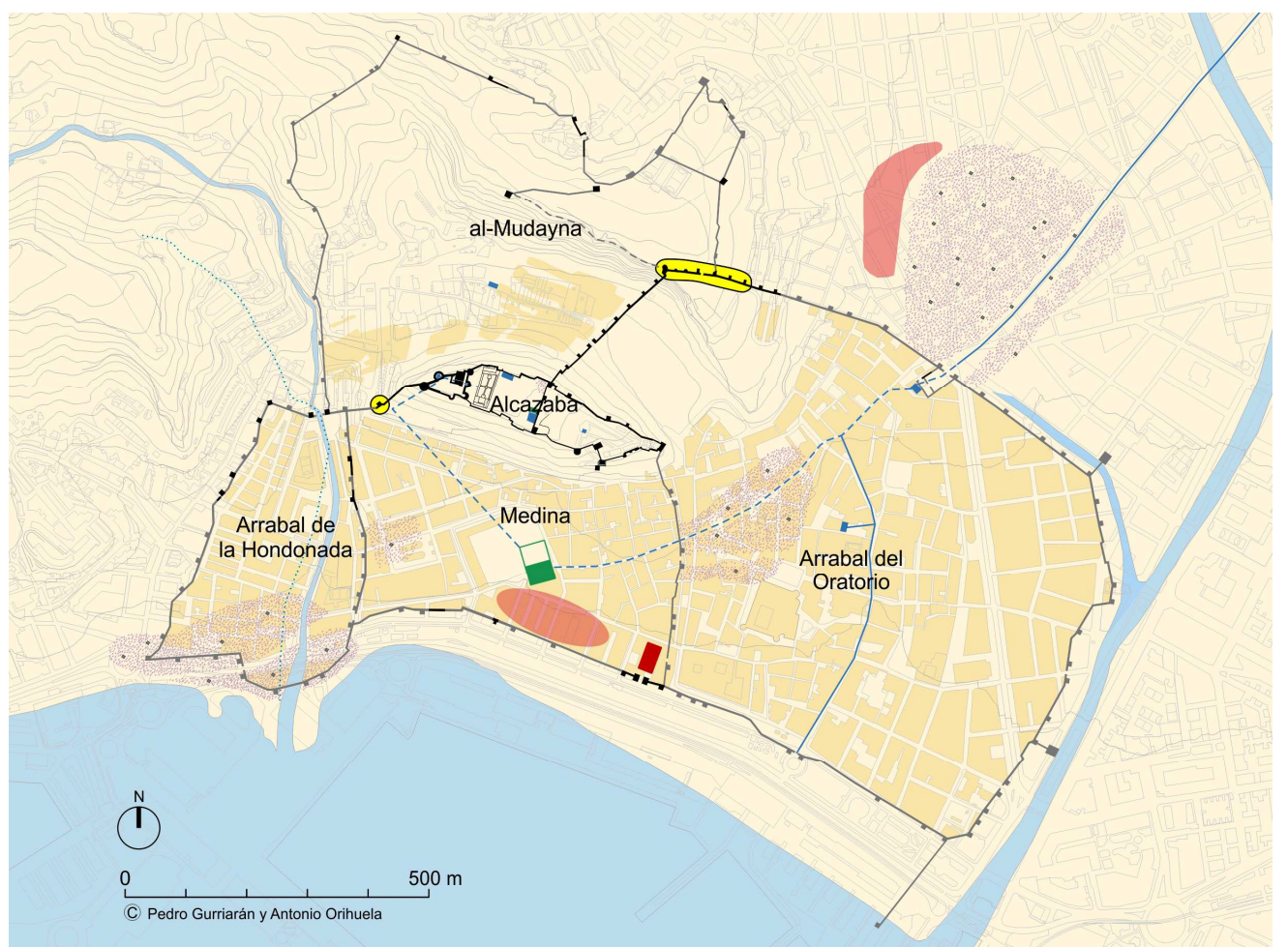

Fig.1 Plano hipotético de Almería en época nazarí, según P. Gurriarán (Yamur, S.L.) y A. Orihuela (EEA, CSIC). Se marca en color amarillo las zonas donde se han tomado las muestras válidas analizadas. 
Por otra parte, en la zona llana del cerro hay cuatro torres con frente redondeado construidas con sillarejo (T1, T2, T3 y T5), que casi todos los investigadores atribuyen a la década de dominio de la coalición cristiana (1147-1157). Entre ellos está la Torre 4, realizada con tapia calicostrada en dos etapas constructivas. En su parte más cercana a los lienzos de la muralla se analizó la Muestra 14 que dio cronología califaltaifa, pero en su parte más saliente la M-15 proporcionó una cronología con probabilidad almohade en un $79 \%$, siendo la altura de cajones en esta zona de $105 \mathrm{~cm}$. Las caras externas de los lienzos intermedios de este sector, que son también de tapia calicostrada, se adosan a las citadas torres de piedra y tienen una altura de cajones de $95 \mathrm{~cm}$. En el lienzo 2-3 la muestra M16 proporcionó una cronología almohade-nazarí inicial (1151-1253) con un 91\% de probabilidad, mientras que la M-13, tomada en el lienzo 4-5, dio como resultado la etapa nazarí con el $100 \%$ de probabilidad (Fig. 2).
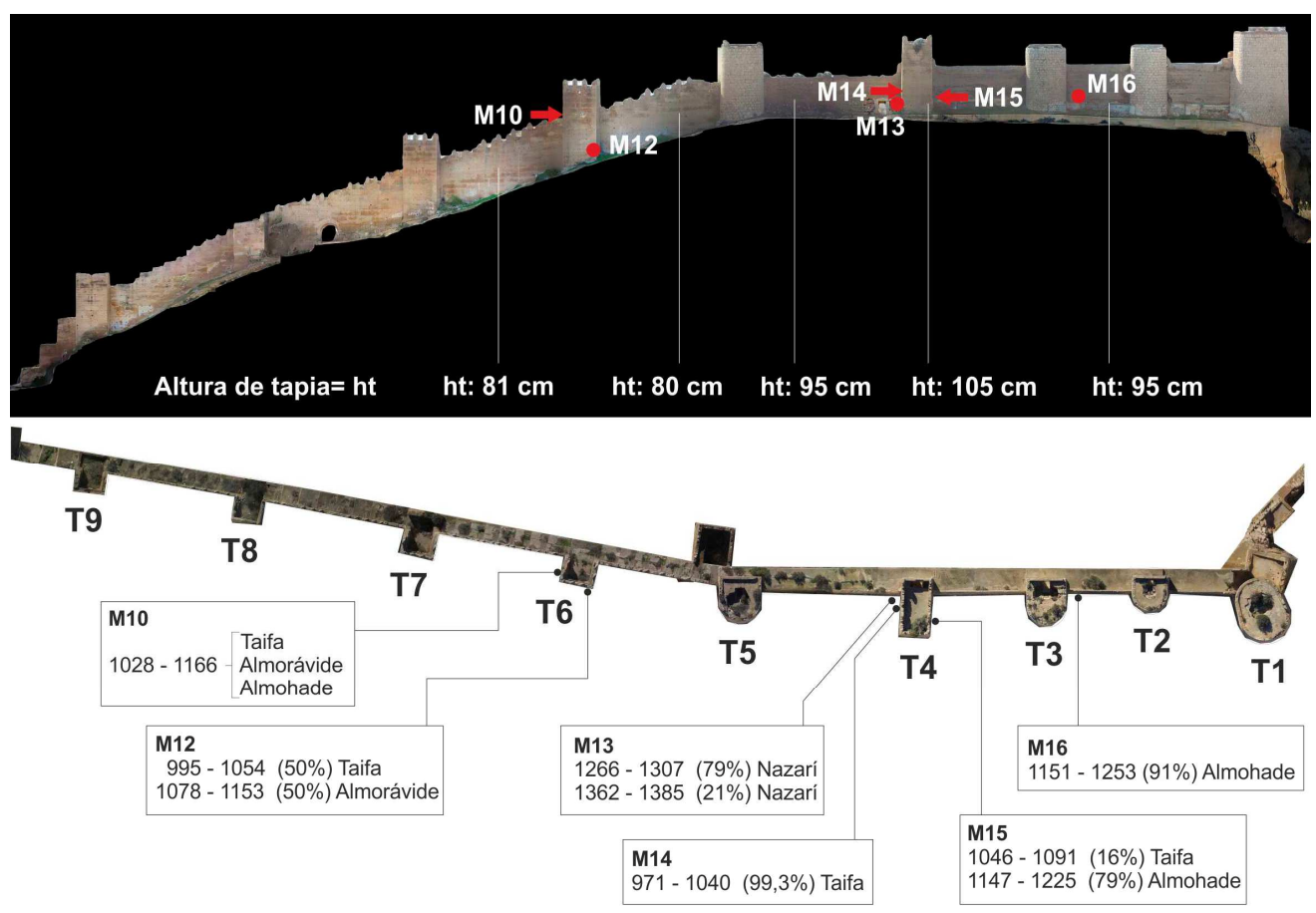

Fig. 2. Planta y alzado extramuros de la muralla del Cerro de San Cristóbal, con datos de ${ }^{14} \mathrm{C}$ y metrológicos (planimetría base de E. Navarro y L. García Pulido, 2017).

En la muralla occidental de la antigua medina, cerca de su inicio en el extremo oeste de la Alcazaba, hay otra torre de tapia calicostrada, de la que pudimos obtener dos muestras de madera (M-17 y M-20) y una de la soga de fibra vegetal (M-18) que sirve para atirantar los encofrados durante el relleno de los mismos con los áridos y aglomerante apropiados. Las tres muestras dan un resultado homogéneo correspondiente a la época nazarí (Fig. 3).

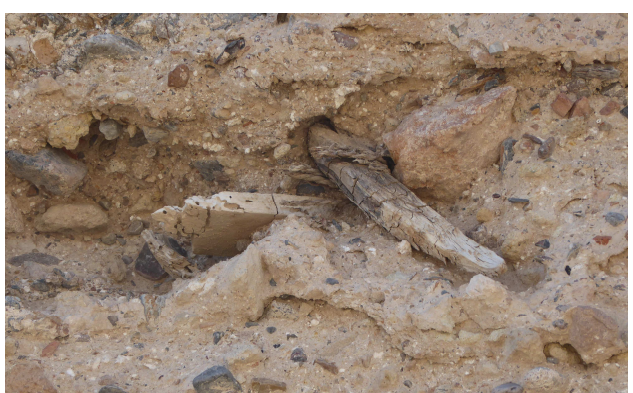

Fig. 3. Aguja, cuña y soga en torre de la Medina. 


\section{Discusión}

Además de los datos proporcionados por los análisis de radiocarbono, las técnicas constructivas y la metrología de las tapias, hemos analizado también las fuentes documentales árabes, las fotografías antiguas y los primeros proyectos de restauración de las murallas en el sector del Cerro de San Cristóbal. Estos proyectos iniciales fueron realizados por el arquitecto conservador responsable de la zona monumental de Andalucía Oriental, Francisco Prieto-Moreno, en los años 1950 (AGA, caja 71.075), 1960 (AGA, caja 70.918), 1961 (AGA, caja 71.183) y por el arquitecto Roberto Puig Álvarez en 1979 (AGA, caja 87.146). De todo ello se puede plantear la hipótesis de que las murallas levantadas bajo el mandato de Jayrān en el Cerro de San Cristóbal durante el primer tercio del siglo XI, debían de estar construidas con tapias de tierra, según el texto que recopiló tres siglos después el geógrafo al-Hịmyarī (m. 1327): "Son faubourg, connu sous le nom d'al-Muṣallā, est entouré d'un rempart de terre (sūr turāb) qui fut élevé par Hayrān le "Āmirite" (al-Ḥimyarī, 1938, p. $221 / \backslash \wedge \mathrm{T}$ ). Por la poca calidad del material utilizado dichas tapias pronto empezarían a deteriorarse.

Pasada la mitad del periodo almorávide en Almería (1091-1147), inmediatamente después de la incursión de Alfonso I de Aragón por el sur de la península ibérica (septiembre 1125 - junio 1126), el emir almorávide 'Alī ibn Yūsuf aumentó el impuesto sobre los edificios, denominado $t a^{\prime} t \bar{t} b$, destinado a reforzar las murallas de las ciudades. En al-Andalus se reforzaron especialmente Granada, Córdoba, Sevilla y Almería. En esta última ciudad "se acabó la muralla, según lo que exigía de fortaleza y hermosura, con el menor gasto" (Ibn 'Id̄ārī, 1963, pp. 170-172). Todos estos datos permiten plantear la hipótesis de que las murallas del Cerro de San Cristóbal fueron reforzadas exteriormente y recrecidas mediante tapias calicostradas de gran calidad. Se trataba de una nueva técnica constructiva en la cual las tapias tienen una costra externa de mortero de cal, realizada en tongadas de unos $10-15 \mathrm{~cm}$ de altura que adquiere gran dureza, mientras que el interior se rellena y apisona con tierra, grava y mampuestos, aglomerados con escasa cal. Con esta misma técnica se reforzaron o ampliaron extensos tramos de murallas en las otras ciudades citadas por Ibn 'Id dārī (Marcos, 2015). Un proceso similar, de refuerzo de murallas de tapias de tierra con otras de tapias calicostradas, se ha constatado arqueológicamente en la ciudad de Murcia y documentalmente en Sevilla y Badajoz, entre otras (Jiménez, 2014, pp. 568-580).

Las fotografías que acompañan a los citados proyectos de restauración redactados por PrietoMoreno muestran la parte intramuros de la muralla del Cerro de San Cristóbal totalmente degradada y desaparecida en algunas zonas. En la parte pendiente, entre las torres 5 y 9 , incluso se puede observar que la losa de hormigón de cal, que constituye el suelo del adarve, se encuentra en voladizo, por haberse desmoronado toda la muralla de tierra inicial (Figs. 4 y 5).

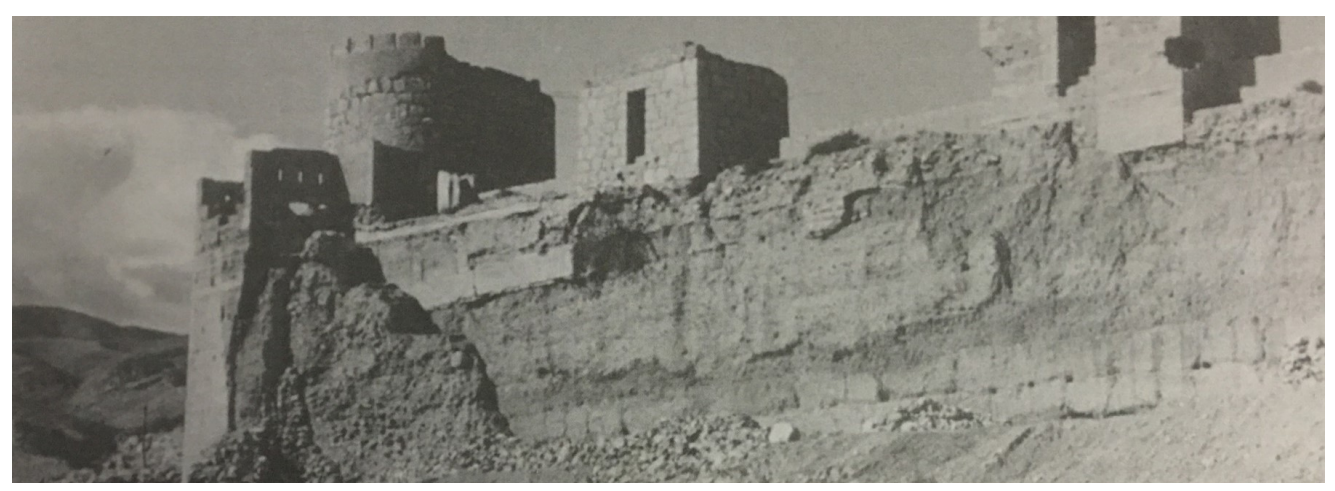

Fig. 4. Muralla del Cerro de San Cristóbal, tramo Torre 1 a Torre 3, vista intramuros. Colección F. Ochotorena, 1955. 


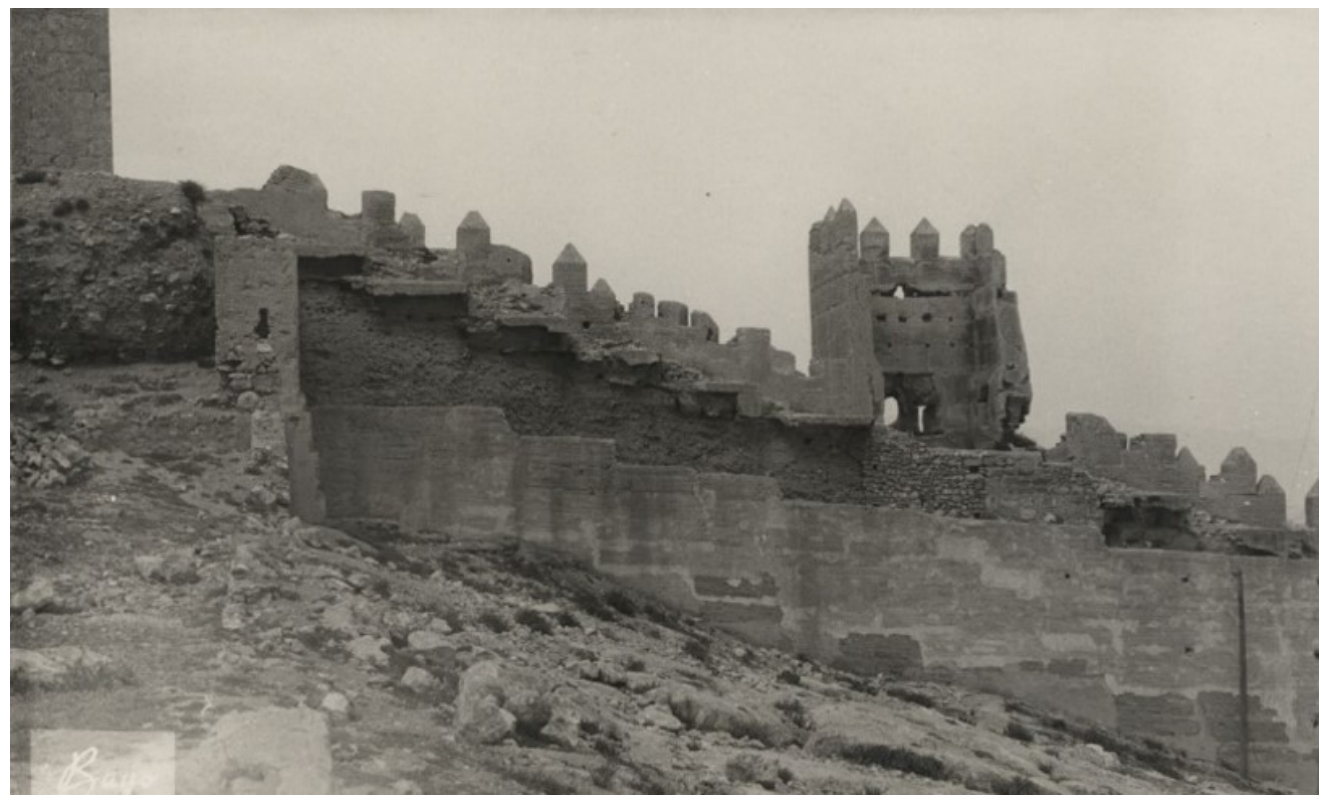

Fig. 5. Muralla del Cerro de San Cristóbal, tramo Torre 5 a Torre 6, vista intramuros, cuando ya se había iniciado la restauración de la parte baja de la muralla (AGA, caja 70.918).

El postigo de San Cristóbal situado entre las torres 4 y 5 constituye un lugar privilegiado para ver en sección las dos fases de esta muralla. En la parte intramuros tiene una anchura de $1,85 \mathrm{~m}$ y conserva sus jambas de excelente fábrica taifa inicial, heredera todavía en este punto singular de la tradición constructiva califal cordobesa con sillares colocados en filas alternativas a soga y tizón. El refuerzo exterior en tapia calicostrada tiene una anchura de $2,03 \mathrm{~m}$, lo que suma un total de 3,88 m. Esta gran anchura solo se da en la parte llana del cerro, entre las torres 1 y 5 , pues entre las torres 5 y 9 la anchura total es menor: 2,60 m. Las torres 6 a 9 y los lienzos situados entre ellas tienen gran similitud con lo conservado tanto en el inmediato sector de la Hoya, como con las torres aisladas conservadas en el sector norte y noroeste de la muralla del Arrabal de la Hondonada (Rabad al-Hawd). La tipología de todas ellas es muy similar y la altura de los cajones de sus tapias también, entre 80-82 $\mathrm{cm}$.

\section{Conclusiones}

Las murallas medievales de Almería sufrieron procesos similares a los ya constatados en otras ciudades de al-Andalus, donde las cercas fundacionales tanto de su medina como de sus arrabales fortificados soportaron intervenciones de reparación, ampliación y/o sustitución de unas murallas fundacionales por otras a lo largo del tiempo. En sus dos arrabales (al-Hawd y alMușallā) las murallas iniciales, que serían de tapias de tierra, fueron ampliadas y/o sustituidas por otras de tapia calicostrada de una gran calidad, lo que ha permitido la conservación de numerosos tramos de lienzos y torres hasta nuestros días. Este proceso debió de producirse en la segunda mitad de la época almorávide, concretamente entre 1126 y 1147 , aunque la técnica constructiva de la tapia calicostrada se siguió manteniendo en sucesivas intervenciones en las etapas almohade y nazarí.

Una evolución similar se puede constatar también en la muralla occidental de la antigua medina, cerca de su inicio en el extremo oeste de la Alcazaba. Los análisis de radiocarbono realizados en la actual torre de tapia calicostrada, que posiblemente sustituyera a una de época califal construida en sillería, dan una cronología homogénea de época nazarí en las tres muestras analizadas. 


\section{Notas}

${ }^{1}$ Este trabajo se ha realizado dentro del marco del Proyecto de I+D del Subprograma Estatal de Generación del Conocimiento: "Las murallas medievales de Almería. Análisis cronotipológico y datación científica" (HAR2015-71609-P), desarrollado entre los años 2016 - 2019, cuyo IP ha sido el Dr. Antonio Orihuela Uzal.
2 Agradezco al Dr. Íñigo Almela Legorburu (LAAC) su ayuda en el análisis de los términos relacionados con los materiales y las técnicas constructivas utilizadas en las murallas en los textos árabes citados.

\section{Bibliography}

al-Ḥimyarī. (1938). La péninsule Ibérique au Moyen-Age d'après le Kitāb ar-Rawḍ al Mi'ṭār, Texte Arabe publié avec une traduction annotée par Lévi-Provençal, E., Leyden.

Alejandre, F.J. (2018). "Consideraciones sobre la datación de morteros de cal mediante ${ }^{14} \mathrm{C}$ ", Revista PH Investigación, 93, pp. 122-140.

Cara, L. (1990). La Almería islámica y su alcazaba, Cajal Ed., Almería.

Garzón, D. (2015). "Almería 1146. Evolución de la estructura urbana y defensas medievales", PH: Boletín del Instituto Andaluz de Patrimonio Histórico, 88, pp. 146-165.

Gil, A. (2014). Almería, la plaza de armas. Más de mil años de fortificaciones, GBG Ed., Almería.

Gurriarán, P; Márquez, S. (2009). "Aparejos constructivos de la Alcazaba de Almería. Lectura, análisis, interpretación", en Suarez, A. coord., Construir en al-Andalus, Monografías del Conjunto Monumental de la Alcazaba, Consejería de Cultura, Junta de Andalucía, Almería, vol. 2, pp. 233-266.

Ibn 'Id̄ārī. (1963). Al-Bayān al-Mugrib. Nuevos fragmentos almorávides y almohades, Huici, A., trad., Valencia.

Jiménez, P. (2014). Murcia: de la Antigüedad al Islam, Universidad de Granada, Granada, in http://hdl.handle.net/10481/31208.

Lirola, J. (1992-93). "Una hipótesis sobre la construcción de la cerca de al-Mudayna en el actual cerro de San Cristóbal (Almería)", Boletín del Instituto de Estudios Almerienses, 11-12, pp. 7-19.

Lirola Delgado, J. (2005). Almería andalusí y su territorio: Textos geográficos, Fundación Ibn Tufayl de Estudios Árabes, Almería.

Marcos, M. (2015). "La huella de Siyilmasa en las cercas almorávides de Marrakech y al-Andalus", Quiroga, 8, pp. $10-22$.

Molina, E. (1986). "Almería islámica: puerta de oriente, objetivo militar (nuevos datos para su estudio en el Kitab iqtibas al-anwar de al-Rusati)", Actas del XII Congreso de la U.E.A.I., Málaga, pp. 559-608.

Navarro, E. (2017). "Estudio detallado del lienzo de muralla de San Cristóbal, en el marco de las fortificaciones medievales de Almería", Trabajo Fin del Master en Rehabilitación Arquitectónica, Universidad de Granada, Granada.

Sánchez, M. (1975-1976). "La cora de Ilbīra (Granada y Almería) en los siglos X y XI, según al-'Uḍī (1003-1085)”, Cuadernos de Historia del Islam, 7, pp. 5-82.

Santos, F.J. (2019). Interpretación del Informe de Datación y Calibración, in http://institucionales.us.es/solicitudes cna/attachments/article/12/Interpretación\%20del\%20Informe\%20de\%20Datación\%20y\%20Calibración.pdf (0112-2019).

Tapia, J.A. (1986). Almería musulmana, edición corregida y aumentada, Cajal Ed.

Torres, L. (1957). "Almería islámica". Al-Andalus, XXII, 2, pp. 411-453. 